\title{
Improved formulation of the coupled-wave method for two-dimensional gratings
}

\author{
Philippe Lalanne \\ Institut d'Optique Théorique et Appliquée, Centre National de la Recherche Scientifique, BP 147, \\ 91403 Orsay Cedex, France
}

Received September 4, 1996; revised manuscript received December 23, 1996; accepted January 21, 1997

A new formulation of the coupled-wave method for two-dimensional gratings is proposed. It is based on mathematical and physical results recently obtained for one-dimensional gratings. Numerical evidence obtained for many different diffraction problems, including dielectric, metallic, volume, and surface-relief gratings, shows that the new formulation outperforms the conventional one in terms of convergence rates. The specific case of gratings with very small thickness, for which opposite conclusions on the convergence performance are obtained, is studied and explained. The methodology can be applied to other numerical techniques that rely on Fourier expansions of the electromagnetic fields and on grating parameters such as the permittivity and the permeability. (C) 1997 Optical Society of America [S0740-3232(97)00108-7]

Key words: coupled-wave method, rigorous grating analysis, diffractive optics.

\section{INTRODUCTION}

Recently, the coupled-wave method for describing the diffraction of electromagnetic waves by one-dimensional (1-D) gratings was revisited. A striking improvement of the convergence performance was reported for TM polarization $^{1,2}$ (magnetic-field vector perpendicular to the grating vector) and for the general conical mounting case. $^{1}$ This improvement was obtained by reformulating the eigenvalue problem in the grating region. The main result reported in Refs. 1 and 2 is that the use of the harmonic coefficients of the relative permittivity or of the inverse relative permittivity has a huge impact on the convergence of the coupled-wave methods. Moreover, as shown in Refs. 1 and 3, it is surprising that the physical intuition gained from asymptotic situations helps one to understand the convergence performance of sophisticated numerical methods. A more mathematical explanation for the improvement in the convergence rate obtained in Refs. 1 and 2 was later given by Li. ${ }^{4}$ More recently, these results have been exploited by $\mathrm{Li}$ and Chandezon ${ }^{5}$ to improve dramatically the convergence performance of the coordinate transformation method $^{6}$ for surface-relief gratings with sharp edges. The net benefit of these works is that, by now, at least two efficient, stable, and versatile theoretical tools are available to model 1-D grating diffraction problems for TE (electric-field vector perpendicular to the grating vector) and TM polarizations and for conical mountings. In my opinion, other rigorous methods too may derive profit from the understanding recently achieved for the coupled-wave method. In this paper, I try to go further and consider two-dimensional (2-D) grating geometries. As rigorous methods for the analysis of $2-\mathrm{D}$ gratings are especially demanding in terms of computational loads, it seems natural to study their modeling in the spirit of the physical and mathematical understanding developed in Refs. 1, 3, and 4. I focus on the coupledwave $\operatorname{method}^{7}$ of 2 -D gratings. A new formulation, di- rectly inspired from the results obtained for the 1-D case, is proposed, and its performance is discussed.

In Section 2 the new system of differential equations is presented. As for the 1-D case, the new formulation does not differ much from the conventional one. Basically, a free parameter $\alpha$ is introduced in the differential equations to weigh the relative strengths of the harmoniccoefficient matrices of the relative permittivity and of the inverse relative permittivity. To show the efficiency of the new formulation, various grating diffraction problems are studied in Section 3. The convergence rates of the two formulations are compared for volume, surface-relief, dielectric, and metallic gratings. Based on the results recently obtained for 1-D gratings, qualitative arguments for understanding the impact of parameter $\alpha$ on the convergence performance are given in Section 4 . For the sake of completeness, the case of thin gratings, for which opposite conclusions are obtained, is reported. It follows from the concluding remarks given in Section 5 that in every case, except for the specific case of very thin gratings, the new formulation outperforms the conventional one in terms of convergence rates and provides an efficient tool for modeling 2-D grating diffraction problems.

\section{NEW EIGENPROBLEM FORMULATION FOR TWO-DIMENSIONAL GRATINGS}

As much has been said about it, the reader is assumed to be familiar with the mathematical formulation of the coupled-wave method. Throughout the paper, notations are those of Refs. 1 and $8 . \quad \mathbf{I}$ is the identity matrix, $\mathbf{K}_{x}$ and $\mathbf{K}_{y}$ are diagonal matrices, $\mathbf{E}$ is a Toeplitz matrix formed by the permittivity harmonic coefficients $\epsilon_{m, n}, \mathbf{A}$ is a Toeplitz matrix formed by the inverse-permittivity harmonic coefficients $a_{m, n}$, and $\mathbf{S}$ and $\mathbf{U}$ are vectors formed by the space harmonic coefficients of the electric and magnetic fields, respectively. The grating structure 
is assumed to be periodic along the $x$ and $y$ directions with periods $\Lambda_{x}$ and $\Lambda_{y}$, respectively. The normal to the grating boundaries is in the $z$ direction. The first derivative in the $z$ variable is denoted by a prime, and $k_{0}$ represents the magnitude of the incident plane-wave vector in vacuum. In the new formulation proposed in this paper, the infinite set of first-order differential equations for the electromagnetic fields is written as

$$
k_{0}{ }^{-1}\left(\begin{array}{c}
\mathbf{S}_{y}^{\prime} \\
\mathbf{S}_{x}^{\prime} \\
\mathbf{U}_{y}^{\prime} \\
\mathbf{U}_{x}^{\prime}
\end{array}\right)=\Omega\left(\begin{array}{c}
\mathbf{S}_{y} \\
\mathbf{S}_{x} \\
\mathbf{U}_{y} \\
\mathbf{U}_{x}
\end{array}\right)
$$

with

$$
\mathbf{\Omega}=\left[\begin{array}{cc}
\mathbf{0} & \mathbf{0} \\
\mathbf{0} & \mathbf{0} \\
\mathbf{K}_{x} \mathbf{K}_{y} & \alpha \mathbf{A}^{-1}+(1-\alpha) \mathbf{E}-\mathbf{K}_{y}{ }^{2} \\
\mathbf{K}_{x}{ }^{2}-\alpha \mathbf{E}-(1-\alpha) \mathbf{A}^{-1} & -\mathbf{K}_{x} \mathbf{K}_{y}
\end{array}\right.
$$

tion tends to unity. In that asymptotic case, the 2-D lamellar grating becomes a 1-D grating with a periodicity along the $x$ direction. From the work of Ref. 1, we know that, in order to yield the fastest convergence rates, the submatrix $\boldsymbol{\Omega}_{2}$ has to be written as

$$
\left[\begin{array}{cc}
\mathbf{K}_{x} \mathbf{K}_{y} & \mathbf{A}^{-1}-\mathbf{K}_{y}{ }^{2} \\
\mathbf{K}_{x}{ }^{2}-\mathbf{E} & -\mathbf{K}_{x} \mathbf{K}_{y}
\end{array}\right]
$$

[ $\alpha=1$ in Eq. (1b)]. Now let us consider the opposite situation, in which the fill factor along the $x$ direction tends to unity. It is easily seen that the submatrix $\boldsymbol{\Omega}_{2}$ has to be written as

$$
\left[\begin{array}{cc}
\mathbf{K}_{x} \mathbf{K}_{y} & \mathbf{E}-\mathbf{K}_{y}{ }^{2} \\
\mathbf{K}_{x}{ }^{2}-\mathbf{A}^{-1} & -\mathbf{K}_{x} \mathbf{K}_{y}
\end{array}\right]
$$

Indeed, second-order differential equations based on Eqs. (1) can be used for effectively reducing the overall computational effort. ${ }^{8,9}$ The square matrix in Eq. (1b) is composed of two submatrices in the upper right and lower left corners, which are denoted by $\boldsymbol{\Omega}_{1}$ and by $\boldsymbol{\Omega}_{2}$, respectively.

In the conventional formulation, which was recently revisited by several authors, ${ }^{8-10}$ the matrix $\Omega$ is

$$
\mathbf{\Omega}=\left[\begin{array}{cccc}
\mathbf{0} & \mathbf{0} & \mathbf{K}_{y} \mathbf{A} \mathbf{K}_{x} & \mathbf{I}-\mathbf{K}_{y} \mathbf{A} \mathbf{K}_{y} \\
\mathbf{0} & \mathbf{0} & \mathbf{K}_{x} \mathbf{A} \mathbf{K}_{x}-\mathbf{I} & -\mathbf{K}_{x} \mathbf{A} \mathbf{K}_{y} \\
\mathbf{K}_{x} \mathbf{K}_{y} & \mathbf{E}-\mathbf{K}_{y}{ }^{2} & \mathbf{0} & \mathbf{0} \\
\mathbf{K}_{x}{ }^{2}-\mathbf{E} & -\mathbf{K}_{x} \mathbf{K}_{y} & \mathbf{0} & \mathbf{0}
\end{array}\right] .
$$

Strictly speaking, there is another conventional formulation. In Ref. 8 the inverse matrix $\mathbf{E}^{-1}$ is used in submatrix $\Omega_{1}$, just as in Eq. (1b). In Refs. 9 and 10 as well as in earlier studies ${ }^{7,11}$ matrix $\mathbf{A}$ is preferred. It should be mentioned that the use of matrix $\mathbf{E}^{-1}$ or $\mathbf{A}$ in submatrix $\boldsymbol{\Omega}_{1}$ has a negligible impact on the convergence performance of both formulations. ${ }^{12}$ For the following comparison, the matrix $\Omega$ in Eq. (2) is used for the coupled-wavemethod implementation of the conventional formulation.

As for the 1-D case, the difference between the new and conventional formulations is rather small. In submatrix $\boldsymbol{\Omega}_{2}$ of the formulation of Eq. (1b), the inverse matrix $\mathbf{A}^{-1}$ is incorporated through a parameter $\alpha$. This parameter is a real positive number in the interval $[0,1]$, which weighs the relative strengths of matrices $\mathbf{E}$ and $\mathbf{A}^{-1}$ in $\boldsymbol{\Omega}_{2}$. To understand the importance of parameter $\alpha$ on the convergence performance (a more technical discussion is given in Section 4), let us consider a 2-D lamellar grating with rectangular grooves. We denote by $f_{x}$ and $f_{y}$ the fill factors along the $x$ and $y$ directions, respectively. The fill factor is defined, along each direction, by the ratio between the groove width and the period. Let us first consider a situation in which the fill factor along the $y$ direc-
[ $\alpha=0$ in Eq. (1b)]. If the adequate submatrices are not used to model these two asymptotic situations, and especially if the conventional formulation of Eq. (2) is used, a slow convergence rate with strong oscillation amplitudes similar to that reported in Fig. 5 of Ref. 1 will be observed. This simple discussion proves that for 2-D gratings, the differential equations of the coupled-wave method should depend on the grating geometry. This is one simple way to understand why parameter $\alpha$ was introduced in the new formulation of Eq. (1b). Two other interpretations for $\alpha$ will be given in Section 4. Finding the optimal value of $\alpha$ that provides the fastest convergence rate for a given grating geometry is a difficult task. However, as will be shown in the next section, a good suboptimal value can be easily derived for simple grating geometries that are generally used in practice.

\section{NUMERICAL EXAMPLES}

In the numerical examples shown hereafter, a linearly polarized electromagnetic wave is obliquely incident at an arbitrary angle of incidence $\theta$ and at an azimuthal angle $\phi$ upon a grating. The angle between the electric-field vector and the plane of incidence is denoted $\psi$. For $\psi=0^{\circ}$ and $\psi=90^{\circ}$, the magnetic and the electric field, respectively, is perpendicular to the plane of incidence. The grating has a thickness $h$ and is surrounded by two semiinfinite media with refractive indices $n_{\mathrm{I}}$ and $n_{\mathrm{II}} \cdot n_{\mathrm{I}}$ and $n_{\text {II }}$ are related to the incident medium and to the substrate, respectively. For numerical purposes the Fourier expansions in the grating region and in the surrounding media are truncated. In the following the truncation is always operated in a symmetric way; the parameter $M$ denotes the highest order retained in the $x$ and $y$ directions ( $-M$ is the lowest order retained). $M$ is called the truncation rank and corresponds to a total of $(2 M+1)^{2}$ retained orders. 
Table 1. Zero-Order Reflected Amplitude Coefficients of a Sinusoidally Modulated Grating ${ }^{a}$

\begin{tabular}{rcc}
\hline$M$ & Conventional Formulation (\%) & New Formulation $(\%)$ \\
\hline 1 & $-68.15+11.45 i$ & $-68.50+10.16 i$ \\
2 & $-68.97+8.30 i$ & $-69.04+7.99 i$ \\
3 & $-69.09+7.73 i$ & $-69.11+7.66 i$ \\
4 & $-69.12+7.60 i$ & $-69.12+7.58 i$ \\
5 & $-69.13+7.57 i$ & $-69.13+7.56 i$ \\
6 & $-69.13+7.56 i$ & $-69.13+7.55 i$ \\
7 & $-69.13+7.55 i$ & $-69.13+7.55 i$ \\
8 & $-69.13+7.55 i$ & $-69.13+7.55 i$ \\
9 & $-69.13+7.55 i$ & $-69.13+7.55 i$ \\
10 & $-69.13+7.55 i$ & $-69.13+7.55 i$ \\
\hline
\end{tabular}

${ }^{a}$ The parameters are $n_{\mathrm{I}}=n_{\mathrm{II}}=1, \epsilon_{0}=16, \Delta \epsilon=15, h=\lambda, \Lambda_{x}$ $=\Lambda_{y}=0.1 \lambda, \theta=\phi=\psi=30^{\circ}$, and $\alpha=0.5$.

The first result concerns a volume grating with a sinusoidal modulation of the relative permittivity:

$$
\epsilon_{r}=\epsilon_{0}+\frac{\Delta \epsilon}{2} \cos \left(2 \pi x / \Lambda_{x}\right)+\frac{\Delta \epsilon}{2} \cos \left(2 \pi y / \Lambda_{y}\right),
$$

with $\Lambda_{x}=\Lambda_{y}$. For this symmetric structure, it is natural to impose the condition that the differential equations (1a) be invariant for the transformations $x \rightarrow y$ and $y \rightarrow x$. Thus the parameter $\alpha$ introduced in the new formulation has to be equal to 0.5. Results of the comparison are shown in Table 1 . The numerical values of the zero-order reflected amplitude coefficients computed with the two formulations are given as a function of the truncation rank. We first observe that, in this simple case, convergence is guaranteed for both formulations for a truncation rank $M=10$, since the exact value of the complex coefficient can be read as $-69.13+7.55 i\left(i^{2}\right.$ $=-1$ ). A careful examination of Table 1 reveals that, whatever the truncation rank is, the new formulation provides better estimates than the conventional one does. Because only nine Fourier coefficients of the relative permittivity are nonzero, this volume grating case is simple and both formulations perform well. Let us now consider more complex situations in which the Fourier expansion of the relative permittivity is slowly converging because of discontinuities.

The second example concerns a lamellar grating with cylindrical grooves and equal periods along the $x$ and $y$ directions. The cylinder diameter is denoted by $D$. Again, for this symmetrical configuration, $\alpha$ is chosen equal to 0.5. Figure 1 shows the zero-order transmitted intensity versus the truncation rank for the two formulations (crosses and circles). Again, a faster convergence rate is obtained with the new formulation. The third example is a lamellar grating with square grooves, which was first studied by Peng and Morris in Ref. 9 because several orders are propagating in the substrate and in the incident medium. On the whole, 14 orders are nonevanescent. As in Fig. 7 of Ref. 9, Fig. 2 shows the diffraction efficiencies of the $(0,0)$ and $(1,0)$ transmitted diffraction orders versus the truncation $\operatorname{rank} M$. Especially for the zeroorder, the convergence rate of the new formulation is excellent: for $4 \leqslant M \leqslant 20$ the zero-order transmitted in- tensities do not differ by more than $0.1 \%$. The fourth example concerns a nonsymmetric grating geometry with parallelepiped grooves. Figure 3 shows the zero-order reflected intensity as a function of the truncation rank. For this nonsymmetric case, the periods along the $x$ and $y$ directions are equal, but the two fill factors strongly dif-

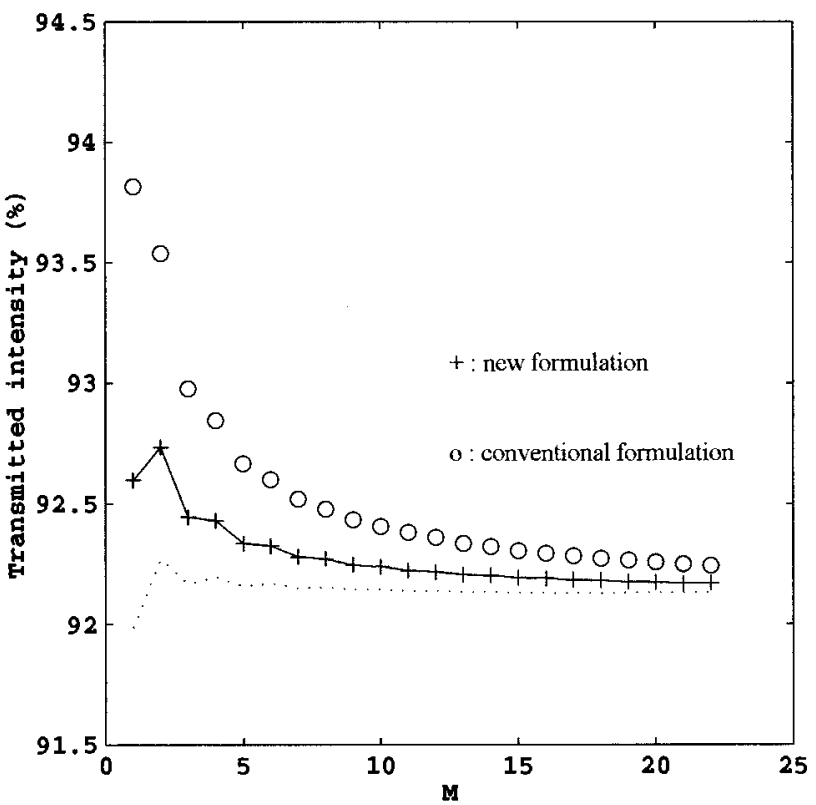

Fig. 1. Zero-order transmitted intensity of a lamellar grating with cylindrical grooves etched in a substrate of refractive index $n_{\mathrm{II}}=2$. Other grating parameters are $n_{\mathrm{I}}=1, h=\lambda, \Lambda_{x}$ $=\Lambda_{y}=0.1 \lambda, D=0.5 \Lambda_{x}, \theta=\phi=0, \psi=90^{\circ}$, and $\alpha=0.5$. The dotted curve is obtained with the new formulation for $\alpha$ $=0.237$ and for an incident plane wave polarized along the $y$ direction.

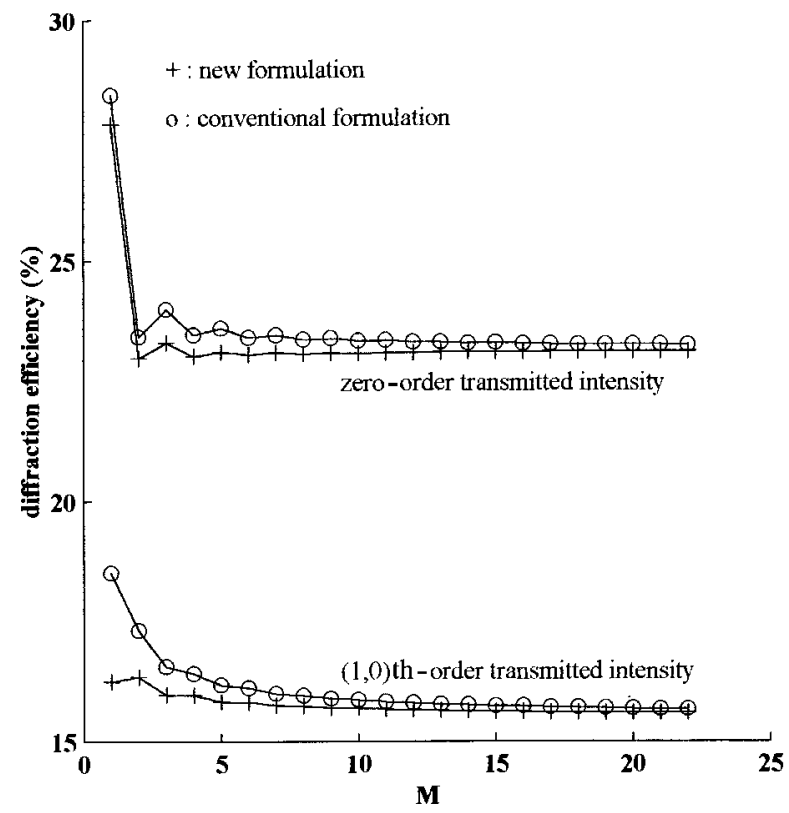

Fig. 2. (0,0) and $(1,0)$ transmitted intensities of a lamellar grating with square grooves etched in a substrate of refractive index $n_{\text {II }}=1.5$. The grating parameters are those given in the caption to Fig. 7 of Ref. 9: $n_{\mathrm{I}}=1, h=\lambda, \Lambda_{x}=\Lambda_{y}=1.2 \lambda, f_{x}$ $=f_{y}=0.5, \theta=\phi=0, \psi=90^{\circ}$, and $\alpha=0.5$. 
fer: $f_{x}=0.1$ and $f_{y}=0.8$. A normally incident plane wave polarized along the $y$ direction is considered, and the parameter $\alpha$ is given by

$$
\alpha=\delta_{f_{y}}\left(1-\frac{\delta_{f_{x}}}{2}\right)+\frac{f_{y} \Lambda_{y}}{f_{x} \Lambda_{x}+f_{y} \Lambda_{y}}\left(1-\delta_{f_{x}}\right)\left(1-\delta_{f_{y}}\right),
$$

where $\delta_{x}=1$ if $x=1$ and 0 if not. The choice of Eq. (3) is not unique and will be discussed in the next section. We just note that according to the above discussion on the introduction of parameter $\alpha$ in Eq. (1b), $\alpha=1$ if $f_{y}=1$, $\alpha=0$ if $f_{x}=1$, and $\alpha=0.5$ if $f_{y}=f_{x}$ and $\Lambda_{x}=\Lambda_{y}$ (symmetric case). In this nonsymmetric case, the best performance in Fig. 3 is achieved for the new formulation. The last example is related to a metallic lamellar grating with square grooves. The incident medium is air, and the substrate is glass $\left(n_{\mathrm{II}}=1.5\right)$. The high and low optical indices of the grating are $n_{h}=(3.18-4.41 i)^{1 / 2}$ and $n_{l}$ $=1$, respectively. So the grooves are metallic and surrounded by air. A comparison of the convergence rates of the two formulations is shown in Fig. 4, where the absorption is plotted versus the truncation rank. Again, a faster convergence rate is obtained with the new formulation.

\section{DISCUSSION}

In every numerical example provided in Section 3, the convergence performance of the new formulation is better than that of the conventional one. Considering, for instance, the numerical results of Figs. 2 and 3, plateaux are obtained with the new algorithm but not with the conventional one. In these two cases, numerical results are more accurate with the new formulation and 289 retained orders $(M=8)$ than with the conventional formulation and 2025 retained orders $(M=22)$. It is worth mentioning that, strictly speaking, retaining 2025 orders in coupled-wave-method computations is far beyond any capability of today's personal computers. Except in the volume grating case, in which a conical diffraction problem was investigated, in every example provided in this paper, symmetry considerations similar to those reported in Ref. 13 were exploited in the code to take advantage of the diffraction-problem degeneracy and to reduce memory requirements. Thus 529 orders, instead of 2025, were actually considered in the computation. Unfortunately, these symmetry arguments do not hold in general. In the recent studies of Refs. 9 and 10, for instance, the maximum truncation ranks used in the tests are only 8 and 12 , respectively. Thus the analysis of $2-\mathrm{D}$ gratings is extremely demanding in computational effort. This is the reason why any convergence improvement like the one reported herein has major practical interest.

As the new algorithm presented in this paper is directly inspired by the 1-D case (see the simple arguments provided in Section 2 for $f_{x}=1$ or $f_{y}=1$ ), it is natural to consider the following question: Can the understanding that we have from the 1-D case be applied to explain the convergence improvement observed for the 2-D case and to choose a good value of parameter $\alpha$ ? To my knowledge, two interpretations are available to explain why the

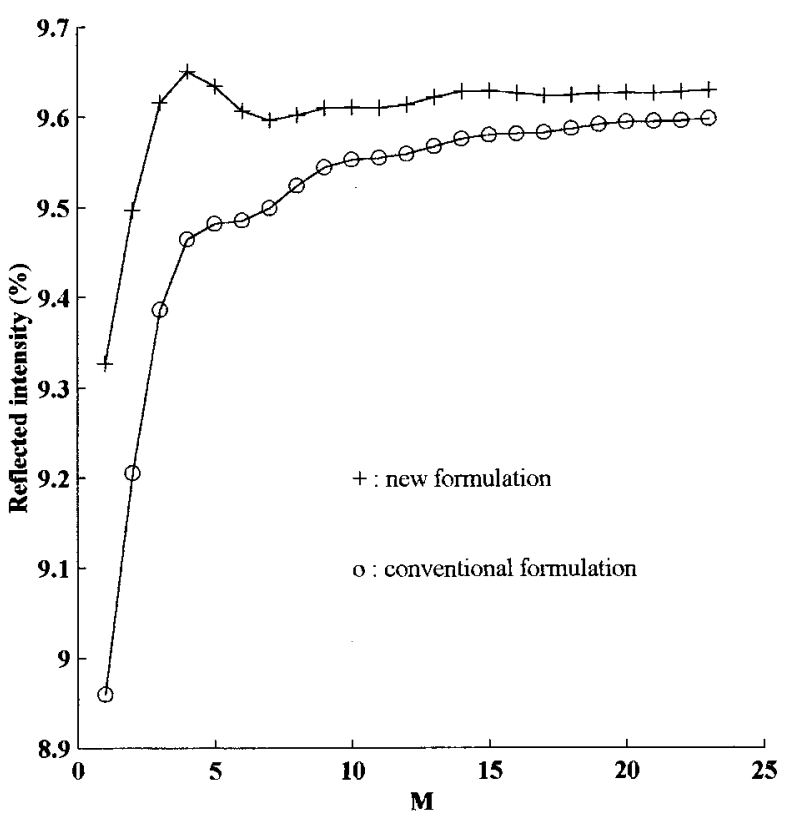

Fig. 3. Zero-order reflected intensity of a nonsymmetric lamellar grating with rectangular grooves etched in a substrate of refractive index $n_{\mathrm{II}}=2$. Other grating parameters are $n_{\mathrm{I}}=1$, $h=\lambda, \Lambda_{x}=\Lambda_{y}=0.1 \lambda, f_{x}=0.1, f_{y}=0.8, \theta=\phi=0$, and $\psi$ $=90^{\circ}$, and $\alpha$ is given by Eq. (3).

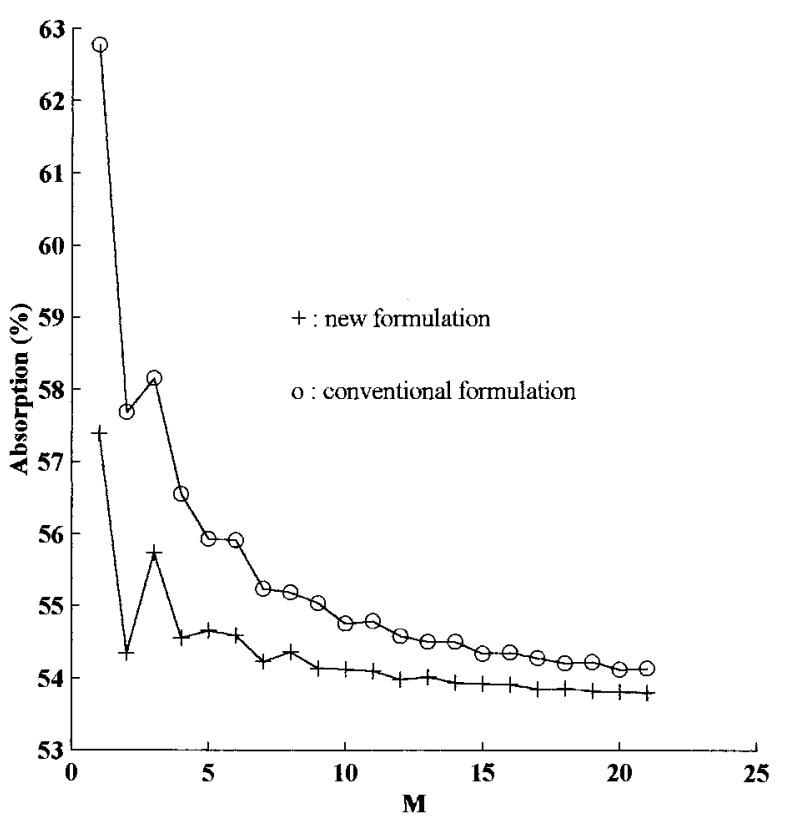

Fig. 4. Absorption of a lamellar grating composed of metallic grooves with refractive index $n_{h}=(3.18-4.41 i)^{1 / 2}$ and deposited on a glass substrate of refractive index $n_{\mathrm{II}}=1.5$. Other grating parameters are $n_{\mathrm{I}}=1, h=0.2 \lambda, \Lambda_{x}=\Lambda_{y}=0.5 \lambda, f_{x}$ $=f_{y}=0.6, \theta=\phi=0, \psi=90^{\circ}$, and $\alpha=0.5$.

use of matrix $\mathbf{E}$ or $\mathbf{A}^{-1}$ strongly influences the convergence performance of the coupled-wave method for 1-D gratings. A physical argument based on the study of the quasi-static limit is given in Ref. 1. The quasi-static limit refers to an asymptotic situation in which the grating period is infinitely small compared with the wavelength. A more mathematical explanation of the perfor- 
mance improvement was given by $\mathrm{Li}^{4}$ for lamellar gratings. Let us first consider how Li's analysis can be applied for 2-D gratings.

\section{A. Boundary Conditions inside the Grating Region}

According to $\mathrm{Li}$, for TM polarization of 1-D gratings, the new formulation converges faster because it uniformly satisfies the boundary conditions in the grating region, whereas the conventional formulation does so nonuniformly. In other words, the new formulation uniformly preserves the continuity of the electromagnetic-field quantities that should be continuous across permittivity discontinuities of lamellar gratings. Let us consider the elementary cell depicted in Fig. 5. It corresponds to a lamellar grating with rectangular grooves. The $y$ component of the electric field is perpendicular and tangential to the $S_{1}$ and $S_{2}$ boundaries, respectively. Following the results of Ref. 4, when writing down the differential equations so as to match uniformly the boundary conditions in the grating, we must consider Laurent's rule (products of type $\mathbf{E S}_{y}$ ) for the $S_{1}$ boundary whereas the inverse rule (products of type $\mathbf{A}^{-1} \mathbf{S}_{y}$ ) has to be considered for the $S_{2}$ boundary. In my opinion, it is not possible to formulate the differential equation of the coupled-wave method in a spatially dependent manner. Thus, except if it is deeply revisited and because of the intrinsic nature of 2 -D gratings, the coupled-wave method cannot be formulated in a way such that boundary conditions are uniformly satisfied in the grating region. Similar conclusions probably hold for the differential ${ }^{14}$ and coordinate transform methods. In general, it is expected that this result also holds for any rigorous method relying on a Fourier expansion of the relative permittivity. However, being aware of the fact that boundary conditions cannot be matched everywhere, we can apply a compromise by trying to match them as much as possible on spatial average. To illustrate our purpose, let us consider again the grating geometry of Fig. 5. In the unit cell, the surfaces of the vertical walls parallel to the $x$ axis and to the $y$ axis are $2 h f_{x} \Lambda_{x}$ and $2 h f_{y} \Lambda_{y}$, respectively. Thus the relative weights of vertical walls parallel to the $y$ axis to the $x$ axis are $u$ $=f_{y} \Lambda_{y} /\left(f_{x} \Lambda_{x}+f_{y} \Lambda_{y}\right)$ and $1-u$, respectively. On spatial average, the $y$ component $\mathbf{S}_{y}$ of the electric field is parallel to a vertical wall with a probability $u$ and perpendicular to a vertical wall with a probability $1-u$. For the $y$ component $\mathbf{S}_{y}$ of the electric-field vector, products of type $\mathbf{E S}_{y}$ and $\mathbf{A}^{-1} \mathbf{S}_{y}$ have to be considered with relative

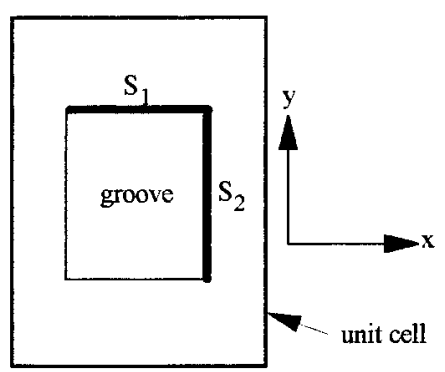

Fig. 5. Unit cell of a 2-D lamellar grating with rectangular grooves. In this top view, the horizontal and vertical lines, $S_{1}$ and $S_{2}$, represent two surface boundaries of the rectangular groove. weights $u$ and $1-u$, respectively. Thus parameter $\alpha$ can be read as $u$. This is the justification of Eq. (3), which is used for the numerical results of Section 3 . Note that the $\alpha$ value defined in Eq. (3) equals $u$ [ $\delta$ signs in Eq. (3) are added just to take into account asymptotic situations for which 2-D gratings become 1-D gratings, i.e., $f_{y}=1$ or $f_{x}=1$ ]. A similar derivation holds for the $x$ component $\mathbf{S}_{x}$ of the electric field. Of course, since the electric field is supposed to be constant over all of the vertical walls, this consideration, which is based on spatial average, is only qualitative and provides only a rough approximation of the optimal value of $\alpha$.

\section{B. Quasi-Static Limit}

Let us now consider how the physical argument of Ref. 1 can be applied for 2-D gratings. In the quasi-static limit, 1-D gratings are strictly equivalent to thin films, and no evanescent orders have to be considered for their modeling. For TM polarization of 1-D gratings, the authors of Ref. 1 showed that with the conventional formulation, an accurate description of the quasi-static limit surprisingly requires that all the evanescent orders are retained in the computation. They concluded that the conventional formulation is badly conditioned. A similar discussion can be found in Ref. 15. On the contrary, as can be expected from the thin-film analogy, the new formulation is well conditioned, since the effective properties are adequately described by retaining only the zero orders. To avoid any confusion with the inverse-problem literature, "bad conditioning" in this paper, as in Refs. 1 and 3, refers to a formulation in which an infinite number of orders is required to model properly a situation (asymptotic limit) that can in principle be modeled by retaining only the zero orders. Similarly, "good conditioning" refers to a formulation in which the zero orders are sufficient to model the situation. In the quasi-static limit, the fundamental mode propagating in a 1-D grating structure is simply a plane wave. For $2-\mathrm{D}$ gratings the mode is no longer a plane wave. ${ }^{13}$ Thus, because of the intrinsic nature of the diffraction problem, the quasi-static limit of 2-D gratings cannot be properly modeled by retaining only the zero order. This result holds for rigorous methods relying on a Fourier expansion of the electromagnetic field inside the grating region.

However, as in the previous subsection, we can apply a compromise. Although the electromagnetic field (eigenmode in the coupled-wave method) cannot be adequately represented by retaining only the zero orders in the grating region, we can impose that the modulus $\beta$ of the wave vector of the fundamental mode (eigenvalue in the coupled-wave method), supported by the periodic structure for a particular linear polarization of the incident plane wave, be accurately computed. As shown in Ref. 1, the convergence performance of a given formulation is deeply related to its capability of providing $\beta$ when retaining only the zero orders. In the quasi-static limit, the ratio $\beta / k_{0}$ is the effective index of the periodic structure. ${ }^{13}$ When only the zero orders in Eqs. (1) are retained, the effective indices $\eta_{x}$ and $\eta_{y}$ for a normally incident plane wave linearly polarized along the $x$ and $y$ directions, respectively, are 


$$
\begin{aligned}
& \eta_{x}(\alpha)=\left[(1-\alpha) \epsilon_{0,0}+\alpha / a_{0,0}\right]^{1 / 2}, \\
& \eta_{y}(\alpha)=\left[\alpha \epsilon_{0,0}+(1-\alpha) / a_{0,0}\right]^{1 / 2} .
\end{aligned}
$$

These expressions can be easily derived by using the methodology developed in Ref. 13. When we retain only the zero orders with the conventional formulation of Eq. (2), the effective indices $\eta_{x}^{\prime}$ and $\eta_{y}^{\prime}$ for a normally incident plane wave linearly polarized along the $x$ and $y$ directions are

$$
\eta_{x}^{\prime}=\eta_{y}^{\prime}=\left(\epsilon_{0,0}\right)^{1 / 2} .
$$

As was noted by Grann et al. ${ }^{16}$ the effective indices $\eta_{x}^{\prime}$ and $\eta_{y}^{\prime}$ obtained with the conventional formulation are strongly inaccurate. With the new formulation of Eq. (1b), parameter $\alpha$ can be seen as a free parameter that can be chosen ${ }^{17}$ such that the effective index coincides with $\beta / k_{0}$ for a particular linear polarization. For the purpose of illustration, let us consider the case of the lamellar grating with cylindrical grooves considered in Section 2 for a normally incident plane wave polarized along the $y$ direction. According to Ref. 13, the effective index in the quasi-static limit is equal to $n_{\text {eff }}=1.130$. This result is obtained for normal incidence and for a truncation rank equal to $30\left(M_{\mathrm{EMT}}=30\right.$ in Ref. 13). With my personal computer, this computation lasts 5 min. According to Eq. (4b), $\eta_{y}$ is equal to $n_{\text {eff }}$ for $\alpha$ $=0.237$. In Fig. 1 the dotted curve represents results obtained with the new formulation for $\alpha=0.237$ and for an incident plane wave polarized along the $y$ direction. Of course, identical results are obtained for $\alpha=0.763$ and a polarization along the $x$ direction. It is noticeable that the new formulation with $\alpha=0.237$ outperforms the conventional one and provides a faster convergence rate than that obtained with the new formulation and $\alpha$ $=0.5$. This is not surprising if we note that the diffraction problem analyzed in Fig. 1 corresponds to a subwavelength grating with a small period-to-wavelength ratio $\left(\Lambda_{x} / \lambda=\Lambda_{y} / \lambda=0.1\right)$. In this case the convergence performance is driven more by quasi-static limit considerations than by arguments based on the uniform matching of the boundary conditions in the grating region.

In the most general formulation, the matrix $\boldsymbol{\Omega}_{2}$ in Eq. (1b) can be written as

$\mathbf{\Omega}_{2}=\left[\begin{array}{cc}\mathbf{K}_{x} \mathbf{K}_{y} & \alpha_{1} \mathbf{A}^{-1}+\left(1-\alpha_{1}\right) \mathbf{E}-\mathbf{K}_{y}{ }^{2} \\ \mathbf{K}_{x}{ }^{2}-\alpha_{2} \mathbf{E}-\left(1-\alpha_{2}\right) \mathbf{A}^{-1} & -\mathbf{K}_{x} \mathbf{K}_{y}\end{array}\right]$,

where $\alpha_{1}$ and $\alpha_{2}$ are two real positive numbers in the interval $[0,1]$. In that case the couple $\left(\alpha_{1}, \alpha_{2}\right)$ may be chosen so that both $\eta_{x}$ and $\eta_{y}$ coincide with the effective index for polarization along the $x$ and $y$ directions. This may be useful when incident plane waves not polarized along either the $x$ or the $y$ direction are considered. This paper is not intended to discuss for what values of $\alpha$ in Eq. (1b) or of $\left(\alpha_{1}, \alpha_{2}\right)$ in Eq. (6) the fastest convergence rate is obtained for a given diffraction geometry. As can be seen from the above considerations, which are driven in terms of effective indices and boundary conditions, this problem is rather difficult and strongly depends on the diffraction problem under consideration. It is preferable to try to learn from one's own experience, starting from a suboptimal value of $\alpha$ that can be derived by simple considerations.

\section{Small-Depth Limit}

In comparison with the strong mathematical argument based on Fourier-series analysis, the simple consideration in the quasi-static limit may appear weak. As was shown for the grating with cylindrical grooves, it is not exact. Also, in some conflicting situations, the conditioning of a given formulation has more impact on the convergence performance than the uniform matching of boundary conditions. As shown in Ref. 3, for 1-D lamellar gratings and for small enough thicknesses, the conventional formulation converges faster than the new one, although the latter provides a uniform matching of the boundary conditions. The same phenomenon occurs for 2-D gratings. For small enough thicknesses, the better performance is obtained with the conventional formulation. This is illustrated in Fig. 6, where the same metallic grating as the one of Fig. 4 is considered for a very small thickness, $h=0.005 \lambda$. Clearly, the worse performance with the largest oscillation amplitudes is obtained with the new formulation of Eqs. (1). This result can be understood by considering that in the small-depth limit ( $h \rightarrow 0$ ), effective indices of 2-D gratings for a normally incident plane wave linearly polarized along the $x$ or the $y$ direction are both equal ${ }^{18}$ to $\left(\epsilon_{0,0}\right)^{1 / 2}$. Their values coincide with those of the conventional formulation of Eq. (5) but not with those of the new formulation of Eqs. (4). More details concerning the specific case of small-depth gratings can be found in Ref. 3, where the convergence rates of the coupled-wave and differential methods are studied for 1-D thin gratings, and in Ref. 18, where the influence of the depth on the effective properties of subwavelength gratings is considered.

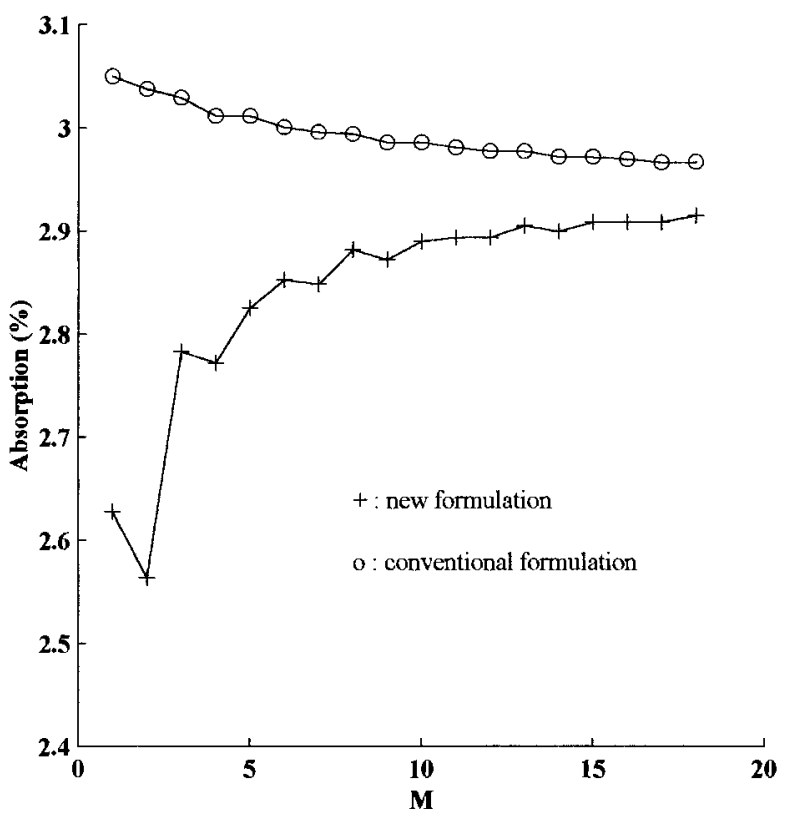

Fig. 6. Same as Fig. 4, except that $h=0.005 \lambda$. 


\section{CONCLUSION}

Through the physical and mathematical understanding recently gained from the study of 1-D grating diffraction problems, the coupled-wave method of 2-D gratings is revisited. Because of the intrinsic nature of 2-D grating diffraction problems, the coupled-wave method cannot be formulated in such a way that the electromagnetic-field quantities that should be continuous across permittivity discontinuities remain so. Also, no formulation can provide an accurate modeling of the electromagnetic field in the quasi-static limit. By the application of a compromise, an improved formulation of the coupled-wave method for 2-D gratings is presented. A new set of firstorder differential equations was proposed for solving Maxwell's equations inside the grating region [see Eqs. (1) and (6)]. Second-order differential equations can be derived simply. The new formulation uses a free parameter $\alpha$ to weigh the relative strengths of matrices $\mathbf{E}$ and $\mathbf{A}^{-1}$ in the differential equations. Several grating diffraction problems are considered to demonstrate the capability of the present method. In every case the new formulation outperforms the conventional one. The exceptional case of very thin gratings, for which opposite conclusions are obtained, is given and explained. The numerical results clearly show the impact of parameter $\alpha$ on the convergence rate of the new formulation. Considerations based on the quasi-static limit and on the continuity of the electromagnetic field in the grating region are given to explain this impact. This interpretation provides not only a physical insight but also a simple way to derive good values of $\alpha$ for some basic grating geometries, including volume or surface-relief, dielectric or metallic gratings, with symmetric or nonsymmetric, round or square grooves. It also suggests that finding the optimal $\alpha^{*}$ value that provides the fastest convergence rate for a given diffraction problem is a difficult task. This is an open question.

The efficiency of the new algorithm makes it possible to model 2-D gratings with an improved accuracy by using a given number of orders, or to save much computation time for a given accuracy. Because 2-D gratings are extremely demanding in computational effort, the convergence improvement achieved herein is of important practical interest. Although the improvement reported herein is significant, the fact that the new formulation neither satisfies the boundary conditions uniformly nor is well conditioned in the quasi-static limit is obviously a drawback that makes it conceptually less complete than its 1-D version. This is due to the nature of the 2-D problem and due to the method itself, which relies on Fourier expansions of the field and the relative permittivity inside the grating region. However, as shown by the numerical examples provided in this paper, the new algorithm can be considered as a versatile and powerful tool to model the diffraction of electromagnetic waves by 2-D gratings. The approach developed in this paper can be generalized for continuous-profile gratings and for stacks of lamellar gratings and can be applied to other rigorous methods that use a Fourier extension of the electromagnetic fields in the grating region.

\section{REFERENCES AND NOTES}

1. Ph. Lalanne and G. M. Morris, "Highly improved convergence of the coupled-wave method for TM polarization," J. Opt. Soc. Am. A 13, 779-784 (1996).

2. G. Granet and B. Guizal, "Efficient implementation of the coupled-wave method for metallic lamellar gratings in TM polarization,” J. Opt. Soc. Am. A 13, 1019-1023 (1996).

3. Ph. Lalanne, "Convergence performance of the coupledwave and the differential methods for thin gratings," J. Opt. Soc. Am. A 14, 1583-1591 (1997).

4. L. Li, "Use of Fourier series in the analysis of discontinuous periodic structures," J. Opt. Soc. Am. A 13, 1870-1876 (1996).

5. L. Li and J. Chandezon, "Improvement of the coordinate transformation method for surface-relief gratings with sharp edges," J. Opt. Soc. Am. A 13, 2247-2255 (1996).

6. J. Chandezon, D. Maystre, and G. Raoult, "A new theoretical method for diffraction gratings and its numerical application," J. Opt. (Paris) 11, 235-241 (1980).

7. M. G. Moharam, "Coupled-wave analysis of twodimensional gratings," in Holographic Optics: Design and Applications, I. Cindrich, ed., Proc. SPIE 883, 8-11 (1988).

8. M. G. Moharam, E. B. Grann, D. A. Pommet, and T. K. Gaylord, "Formulation for stable and efficient implementation of the rigorous coupled-wave analysis of binary gratings," $\mathrm{J}$. Opt. Soc. Am. A 12, 1068-1076 (1995).

9. S. Peng and G. M. Morris, "Efficient implementation of rigorous coupled-wave analysis for surface relief gratings," J. Opt. Soc. Am. A 12, 1087-1096 (1995).

10. E. Noponen and J. Turunen, "Eigenmode method for electromagnetic synthesis of diffractive elements with threedimensional profiles," J. Opt. Soc. Am. A 11, 2494-2502 (1994).

11. S. T. Han, Y. L Tsao, R. M. Walser, and M. F. Becker, "Electromagnetic scattering of two dimensional surface-relief dielectric gratings," Appl. Opt. 31, 2343-2352 (1992).

12. This was confirmed by numerical computation results. Also, it can be understood by considering the work of Ref. 4 and by noting that the submatrix $\boldsymbol{\Omega}_{1}$ operates over continuous variables, namely, the magnetic-field vectors.

13. Ph. Lalanne and $\mathrm{D}$. Lemercier-Lalanne, "On the effective medium theory of subwavelength periodic structures," J. Mod. Opt. 43, 2063-2085 (1996).

14. M. Nevière, P. Vincent, and R. Petit, "Sur la théorie du réseau conducteur et ses applications à l'optique," Nouv. Res. Opt. 5, 65-77 (1974).

15. J. Turunen, "Form-birefringence limits of Fourierexpansion methods in grating theory," J. Opt. Soc. Am. A 13, 1013-1018 (1996).

16. E. B. Grann, M. G. Moharam, and D. A. Pommet, "Artificial uniaxial and biaxial dielectrics with use of two-dimensional subwavelength binary grating," J. Opt. Soc. Am. A 11, 2695-2703 (1994).

17. It can be shown that, in the quasi-static limit, $\beta / k_{0}$ is larger than $1 / a_{0,0}$ and smaller than $\epsilon_{0,0}$. Thus, for any arbitrary grating geometry, a solution for $\alpha$ of $\eta_{x}(\alpha)=\beta / k_{0}$ [or $\left.\eta_{y}(\alpha)=\beta / k_{0}\right]$ exists in the interval $[0,1]$. For more details see S. R. Coriel and J. L. Jackson, "Bound on transport coefficients of two-phase materials," J. Appl. Phys. 39, 47334736 (1968).

18. Ph. Lalanne and D. Lemercier-Lalanne, "Depth dependence of the effective properties of subwavelength gratings," J. Opt. Soc. Am. A 14, 450-458 (1997). 